\title{
Avaliabilidade do Projeto Vivências e Estágios na realidade do Sistema Único de Saúde
}

\author{
Evaluability of the Experiences and Internships Project in the Reality of the Unified Health System \\ Evaluabilidad del Proyecto de Experiencias y Pasantías en la Realidad del Sistema Único de Salud
}

Recebido: 11/03/2021 | Revisado: 18/03/2021 | Aceito: 18/03/2021 | Publicado: 27/03/2021

Eduardo Carvalho de Souza

ORCID: https://orcid.org/0000-0002-2494-1177 Universidade de Ribeirão Preto, Brasil

E-mail: eduardo_carvalho21@ @otmail.com

José Maria Ximenes-Guimarães

ORCID: https://orcid.org/0000-0002-5682-6106 Universidade Estadual do Ceará, Brasil E-mail: jose.ximenes@uece.br

Maristela Inês Osawa Vasconcelos ORCID: https://orcid.org/0000-0002-1937-8850

Universidade Estadual Vale do Acaraú, Brasil E-mail: miosawa@gmail.com

Maria do Socorro de Sousa

ORCID: https://orcid.org/0000-0002-1009-0973 Universidade Federal do Ceará, Brasil E-mail: sousams3@gmail.com

Maria Rocineide Ferreira da Silva ORCID: https://orcid.org/0000-0002-6086-6901 Universidade Estadual do Ceará, Brasil E-mail: rocineideferreira@gmail.com

Carlos Garcia Filho

ORCID: https://orcid.org/0000-0002-0345-6033 Universidade de Fortaleza, Brasil E-mail: cgarciafilho@gmail.com

Patrícia Freire de Vasconcelos

ORCID: https://orcid.org/0000-0002-6158-9221 Universidade da Integração Internacional da Lusofonia Afro-Brasileira, Brasil E-mail: patriciafreire@unilab.edu.br

\begin{abstract}
Resumo
Objetivo: Analisar a avaliabilidade do Projeto Vivências e Estágios na Realidade do Sistema Único de Saúde (VERSUS). Metodologia: Estudo de Avaliabilidade, com abordagem qualitativa, realizado no estado do Piauí. Procedeu-se análise de documentos oficiais do VER-SUS, entrevistas com 32 atores envolvidos na execução do projeto e construção de consensos nos resultados da avaliabilidade. O material empírico foi submetido à análise de conteúdo temática. Resultados: Foram identificados os objetivos e metas do Projeto VER-SUS, bem como sua operacionalização no estado do Piauí, o que subsidiou a sistematização do Modelo Lógico. Este foi submetido à apreciação dos atores entrevistados que participaram da execução do projeto, estabelecendo-se um consenso em relação aos pressupostos, à coerência interna e o problema ser resolvido pelo projeto, considerando-se os resultados esperados e impactos. Considerações finais: Os resultados demonstraram que o VER-SUS é passível de avaliação, pois o modelo lógico permitiu a identificação de dimensões que se constituem focos prioritários de processos avaliativos voltados a aferir sua efetividade, cujo problema principal é a formação de profissionais críticos e sensíveis às necessidades da população e ao fortalecimento do SUS.
\end{abstract}

Palavras-chave: Avaliação em saúde; Ensino; Profissionais de saúde; Sistema Único de Saúde; Estágios.

\begin{abstract}
Objective: To analyze the evaluability of the Experiences and Internships Project in the Reality of the Unified Health System (VER-SUS). Methodology: Evaluability study, with a qualitative approach, carried out in the state of Piauí. Analysis of official VER-SUS documents was carried out, interviews with 32 actors involved in the execution of the project and construction of consensus on the results of the evaluability. The empirical material was subjected to thematic content analysis. Results: The objectives and goals of the VER-SUS Project were identified, as well as its operationalization in the state of Piauí, which subsidized the Logical Model systematization. This was submitted to the appreciation of the actors interviewed who participated in the execution of the project, establishing a consensus in relation to the assumptions, internal coherence and the problem to be solved by the project, considering the expected
\end{abstract}


results and impacts. Conclusion: The results showed that VER-SUS is subject to evaluation, since the logical model allowed the identification of dimensions that constitute priority focuses of evaluation processes aimed at assessing their effectiveness, whose main problem is the formation of critical and sensitive professionals. needs of the population and the strengthening of the Unified Health System.

Keywords: Health evaluation; Teaching; Health professionals; Unified Health System; Internships.

\section{Resumen}

Objetivo: Analizar la evaluabilidad del Proyecto Experiencias y Pasantías en la Realidad del Sistema Único de Salud (VER-SUS). Metodología: Estudio de evaluabilidad, con enfoque cualitativo, realizado en el estado de Piauí. Se realizó análisis de documentos oficiales VER-SUS, entrevistas con 32 actores involucrados en la ejecución del proyecto y construcción de consensos sobre los resultados de la evaluabilidad. El material empírico se sometió a análisis de contenido temático. Resultados: Se identificaron los objetivos y metas del Proyecto VER-SUS, así como su operacionalización en el estado de Piauí, que subsidió la sistematización del Modelo Lógico. Este fue sometido a la apreciación de los actores entrevistados que participaron en la ejecución del proyecto, estableciendo un consenso con respecto a los supuestos, coherencia interna y el problema a ser resuelto por el proyecto, teniendo en cuenta los resultados e impactos esperados. Conclusión: Los resultados demostraron que VER-SUS está sujeto a evaluación, ya que el modelo lógico permitió identificar dimensiones que constituyen focos prioritarios de los procesos de evaluación orientados a valorar su efectividad, cuyo principal problema es la formación de profesionales críticos y sensibles a las necesidades de la población y el fortalecimiento del SUS.

Palabras clave: Evaluación de la salud; Enseñanza; Profesionales de la salud; Sistema Único de Salud; Pasantías.

\section{Introdução}

A implantação do Sistema Único de Saúde (SUS), com proposta de reorientação e reorganização dos serviços com vistas à mudança do modelo de atenção, orientado pela integralidade, equidade e humanização, requisita mudanças das práticas dos profissionais de saúde. Nesse contexto, evidencia-se um descompasso entre o mundo da formação e o mundo do trabalho, entre ensino na saúde e as necessidades da população brasileira, expresso no desenvolvimento de um perfil profissional que ainda mostra-se insuficiente para um trabalho em saúde em que se reconheça adequadamente a determinação social do processo saúde-doença, tal como para a produção do cuidado integral, equânime e com resolubilidade.

O modelo hegemônico de formação dos profissionais de saúde, pautado no modelo biomédico, cuja abordagem clássica do ensino é predominantemente a transmissão de informação e o treinamento prático em procedimentos terapêuticos e diagnósticos, com ênfase na especialidade, gerando fragmentação dos processos de cuidado, tem se mostrado insuficiente para preparar as novas gerações de profissionais para atuar no contexto atual do trabalho em saúde. Ainda se observa baixo impacto desta atuação profissional na alteração dos indicadores de saúde de coletividades, insatisfação dos usuários e práticas iatrogênicas descomprometidas com as necessidades de saúde da população (Pinheiro \& Ceccim, 2011; Vendruscolo, Prado \& Kleba, 2016).

Com efeito, tal modelo mostra-se incoerente em relação à operacionalização do Sistema Único de Saúde (SUS) e às propostas estabelecidas pelas Diretrizes Curriculares Nacionais (DCN), que estão direcionadas para uma formação voltada a atender às necessidades do sistema de saúde, em que deve haver a fusão entre a teoria e a prática, o trabalho em equipe multiprofissional, a integralidade da atenção à saúde com corresponsabilização e estabelecimento de vínculo, assim como a parceria entre os mundos do trabalho e da formação profissional (Guimarães, Garcia Filho \& Ruiz, 2019).

Esse panorama tem suscitado reflexão crítica e intensos debates em todas as profissões da saúde, sobretudo em relação à urgência em reorientar a formação e, consequentemente, às práticas de cuidado. Desse modo, tem-se proposto a estruturação de práticas de ensino cujo processo de planejamento do processo ensino-aprendizagem considere as necessidades dos estudantes e profissionais, a centralidade nos problemas de saúde da população, bem como a ampliação dos cenários de aprendizagem, o que requisita efetivar a articulação entre o mundo da formação e o mundo do trabalho na saúde, materializada na integração ensino-serviço-comunidade (Vendruscolo et al., 2016; Mendes et al., 2020). 
No enfretamento dessa problemática, tem-se observado a articulação entre os Ministérios da Educação e da Saúde, com vistas ao desenvolvimento de propostas e estratégias que viabilizem a execução da política de formação da força de trabalho em saúde, o que configura condição necessária à consolidação do SUS. Dessa forma, cumpre o mandato constitucional, estabelecida na Carta Magna de 1988, segundo o qual compete ao SUS ordenar a formação de recursos humanos na área da saúde (Costa, Silva, Lima, \& Ribeiro, 2018; Guimarães et al., 2019).

Não obstante, tem-se encontrado desafios e resistência na implantação das DCN dos cursos da área de saúde. Nesse sentido, identificam-se aversões provenientes de parte dos docentes, que veem a proposta de integração ensino-serviçocomunidade como uma ameaça à importância atribuída às disciplinas e ao hospital universitário e seus ambulatórios de especialidades como único local privilegiado de ensino (Stella \& Puccini, 2008). Ademais, observa-se, também, a dificuldade em trabalhar a formação crítico-reflexiva, a interdisciplinaridade e a humanização (Moraes, Vieira \& Campos, 2017). Reconhece-se, no entanto, que a formação em saúde deve superar essas barreiras e ampliar as condições de atendimento às necessidades de saúde das pessoas e das populações, da gestão setorial, da atenção à saude e do controle social em saúde (Feuerwerker, 2014).

No contexto universitário, dentre as estratégias adotadas para reorientação do ensino de graduação na área da saúde, pode-se apontar o Programa Nacional de Reorientação da Formação Profissional em Saúde (Pró-Saúde) e o Programa de Educação pelo Trabalho para a Saúde (PET-Saúde), ambos com o intuito de fomentar a aprendizagem com base na realidade concreta do trabalho em saúde, promovendo a inserção do aluno nos serviços do SUS, que passam a se constituir cenários de aprendizagem (Guimarães et al., 2019; Lima et al., 2019).

Além destas, insere-se também o Projeto Vivências e Estágios na Realidade do Sistema Único de Saúde (VER-SUS) que se destaca como estratégia que possibilita aos estudantes a oportunidade de conhecerem e experimentarem a realidade do SUS, considerando seu cotidiano, avanços e desafios, como potente cenário de aprendizagem. Estruturado no formato de imersão, por meio da qual o grupo de estudantes é inserido no cotidiano dos serviços de saúde, permite a observação do trabalho das equipes de saúde e da organização dos serviços, potencializando a formação de compromisso ético-político com o processo de construção do SUS, tal como o amadurecimento para a prática interprofissional e para a integração ensinoatenção-gestão-controle social na saúde, conhecido como quadrilátero da formação em saúde (Maranhão \& Matos, 2017; Ferla, Ramos \& Leal, 2013). Essa inserção/imersão de estudantes de várias áreas do conhecimento na gestão e participação social do SUS promove uma formação diferenciada, na qual os sujeitos passam a compreender muito além da organização e operacionalização do sistema, como também a perceber sob uma ótica mais transparente as relações envolvidas no cuidado, na gestão e nos processos de trabalho em que estarão inseridos como profissionais.

O Projeto VER-SUS considera como substrato para seu desenvolvimento as transformaç̃os sociais, assim como seu impacto na reflexão sobre a mudança do currículo mínimo, as formas de ensino-aprendizagem, dentre outros aspectos. Constata-se, ainda, o fato de que essa proposta de vivência e estágio está extremamente articulada ao que se propõe como alternativa para avaliar e intervir na formação acadêmica (Mendes, Fonseca, Brasil \& Dalbello-Araújo, 2012). Tem uma envergadura nacional com um escopo estabelecido pela Associação Brasileira Rede Unida (executora) em parceria com o Ministério da Saúde (financiadora), além de dispor da colaboração dos executores das atividades, que são: Movimento Estudantil; Instituições de Ensino Superior; Gestores; Profissionais de saúde; Controle social, dentre outros (Ferla et al., 2013).

Essas parcerias firmadas corroboraram para o crescimento significativo do Projeto no Brasil, dado o maior quantitativo de alunos e instituições envolvidas. Nesse contexto, a gestão do projeto respeita as especificidades locais no seu modo organizacional, o que em certa medida pode aparentar uma desarticulação entre as diversas vivências que acontecem simultaneamente, por não terem programações engessadas e impostas para todos os coletivos do projeto. 
Dado este crescimento é importante se pensar em um processo avaliativo em todas as dimensões que integram a operacionalização do projeto, principalmente com o intuito de evidenciar as potencialidades e desafios existentes e, com isso, se necessário, articular linhas de fuga para que se possa proporcionar um maior impacto do projeto, colaborando assim com seu alicerçamento e com a formação em saúde para a consolidação do SUS.

Embora alguns estudos venham apontando as potencialidades e contribuições do VER-SUS na reorientação da formação dos profissionais de saúde (Maranhão \& Matos, 2017; Fettermann et al., 2018), é oportuno reconhecer que sua operacionalização não é permanente, não existe uma regularidade, as experiências são pontuais e induzidas por editais. Com isso, é relevante avaliar o projeto até mesmo como estratégia para seu reordenamento e organização, considerando seu potencial de expansão e consolidação, tal como para inferir evidências que comprovem suas contribuições para a formação a fim de buscar garantir os recursos financeiros ministeriais.

Ademais, ainda não se tem evidências da avaliabilidade do projeto em relação à sua operacionalidade e alcance dos objetivos propostos, bem como acerca de quais são as suas potencialidades e obstáculos na reorientação da formação em saúde. Assim, levantou-se o seguinte questionamento: Quais os aspectos conformam o modelo lógico do Projeto VER-SUS no Estado do Piauí? Desse modo, tem-se por objetivo analisar a avaliabilidade do Projeto Vivências e Estágios na Realidade do Sistema Único de Saúde no Piauí.

\section{Metodologia}

Trata-se de um estudo de avaliabilidade (EA), de cunho exploratório e abordagem qualitativa, orientado pelos pressupostos teórico-metodológicos de Thurston e Ramaliu (2005). Configura-se como um estudo pré-avaliativo, que compreende um conjunto de etapas que precedem a avaliação, com vistas a maximizar seu uso, a potencialidade e a oportunidade dos processos avaliativos (Vieira-da-Silva, 2018).

A pesquisa foi desenvolvida no estado do Piauí, com o propósito de realizar estudo de avaliabilidade do Projeto VERSUS, abrangendo os quatro coletivos envolvidos nas ações inerentes à sua implementação, situados em Teresina, Parnaíba, Picos e Floriano. As comissões organizadoras do VER-SUS são compostas por docentes e discentes das graduações da área da saúde, representantes da gestão, trabalhadores do SUS e controle social. Com base no modelo proposto por Thurston e Ramaliu (2005), desenvolveu-se as seguintes fases: identificação de metas, objetivos e atividades da intervenção; Definição dos potenciais envolvidos; Identificação e delineamento da intervenção - construção do Modelo Lógico (ML).

Para operacionalização do estudo, realizou-se análise documental, centrada nos seguintes documentos: editais do projeto; guia do facilitador, cadernos de textos e relatórios de vivências. Com efeito, os documentos foram analisados com foco nos seguintes aspectos: (1) presenças de objetivos; (2) organização do projeto; (3) público-alvo; (4) ações necessárias à execução das vivências; (5) metas a serem alcançadas; e, (6) resultados esperados.

Foram realizadas entrevistas com 32 participantes, considerados informantes-chave, dada a sua implicação na execução do VER-SUS. Esses foram representados por: 16 viventes - designação conferida aos estudantes; oito facilitadores atores que já experienciaram o VER-SUS, com participação em movimento estudantil, ou movimentos sociais, ou extensão universitária no SUS; oito membros de comissão organizadora - docentes, gestores e controle social dos municípios de execução desse projeto. As entrevistas semiestruturadas, audiogravadas e transcritas na íntegra, foram mediadas por um roteiro norteador, contendo perguntas referentes ao conhecimento dos objetivos e à operacionalização das vivências no SUS.

Na perspectiva de apresentar e validar o Modelo Lógico entre os participantes, buscou-se a construção de consensos sobre o resultado da avaliabilidade. Para tanto, foi elaborada uma proposta inicial, com base na análise das informações procedentes dos documentos e das entrevistas, submetida à apreciação dos entrevistados, que foram convidados a empreender 
críticas e apontar sugestões, conforme seu acúmulo teórico-prático conferido pela inserção nas vivências no SUS. Desse modo, os ajustes sugeridos foram incorporados na matriz final do Modelo Lógico do Projeto VER-SUS.

Destarte, para todos os participantes do estudo, foram adotados os seguintes critérios de inclusão: ter participado de pelo menos uma edição do projeto, ter disponibilidade para conceder entrevista, sendo incluso somente em uma das categorias dos sujeitos propostas para o estudo, visto que algumas pessoas já participaram de diversas vivências em categorias diferentes. Como critério de exclusão foi considerado a não participação do Projeto VER-SUS no estado do Piauí.

De posse do material empírico, procedente dos documentos e das entrevistas, procurou-se organizá-lo em categorias, que foram analisadas com base na análise de conteúdo temática, conforme Minayo (2014), condensando-se, nos núcleos de significados/sentidos, os trechos relacionados às questões específicas do estudo.

Esta pesquisa foi aprovada pelo Comitê de Ética em Pesquisa da Universidade Estadual do Ceará (UECE) com o número do Parecer 1.736.260. Todos os participantes assinaram o Termo de Consentimento Livre e Esclarecido.

\section{Resultados e Discussão}

\section{Contexto político-organizacional da implementação do VER-SUS}

O desenvolvimento de estudos de avaliabilidade mostra-se relevante por permitir a identificação das necessidades de conhecimento e de informação das partes interessadas, além de explicitar o contexto político-organizacional da intervenção ao se extrair a realidade social na qual o projeto é executado, assim como a forma como é percebido por seus executores (Leviton, Khan, Rog, Dawkins, \& Cotton, 2010).

Desde sua implantação, o SUS se depara com o desafio da reorientação da formação e da qualificação dos profissionais de saúde, cujo enfrentamento ocorre gradativamente na proposição de políticas de gestão e formação da força de trabalho em saúde. Dessa maneira, o Projeto VER-SUS se constitui em mais uma estratégia voltada a solucionar esta necessidade ao promover ampliação das possibilidades de formação dos profissionais de saúde de modo coerente com as necessidades de saúde da população, comprometidos com a integralidade e qualidade da atenção, além da defesa do direito à saúde fomentando a participação e o controle social.

Com efeito, a análise documental permitiu identificar que o VER-SUS constitui-se como uma intervenção voltada ao enfrentamento dos seguintes problemas na formação dos profissionais de saúde: 1) Descompasso entre os processos de formação centrados na clínica e em treinamentos teórico-práticos de diagnósticos e terapêuticas individuais; 2) Cenários de aprendizagem centrados na sala de aula e hospitais universitários; 3) Desarticulação dos processos de ensino-aprendizagem com a realidade do mundo do trabalho em saúde; Descompromisso ético-político dos profissionais com a consolidação do SUS, com baixa capacidade de incentivar a participação e controle social; 4) Dificuldade de articulação intersetorial e interinstitucional para a integração ensino-serviço-gestão-controle social em saúde; 5) Baixa capacidade de desenvolver a formação para o trabalho em equipe, na perspectiva da interdisciplinaridade.

A proposta de implementação do VER-SUS resulta de uma parceria entre o Ministério da Saúde, a Associação Brasileira Rede Unida e outros colaboradores. A Rede Unida, por sua vez, é responsável pela Secretaria Executiva VER-SUS, atuando na expansão da articulação e produção conjunta sobre temas relacionados às políticas de educação e saúde, com projetos de integração abrangendo instituições de ensino, sistemas de saúde e movimentos sociais, assim como a formação profissional para a área de saúde (Ferla et al., 2013; Maranhão \& Matos, 2017).

No âmbito do estado do Piauí, a realização da primeira edição do VER-SUS ocorreu em 2004, com a organização centrada no Núcleo de Estudos em Saúde Pública (NESP), da Universidade Federal do Piauí (UFPI), com execução somente na capital, Teresina. 
A gente[...] fez o VER-SUS Teresina em 2004, duas vezes. [...] depois eu acho que não teve, parou o financiamento [...]. Ele foi reestruturado e, se eu não tiver enganado, [...] em 2011-2012 retorna nacionalmente o projeto [...] Então a gente recebe o convite, disparado agora pela coordenação nacional que se insere lá na Rede Unida, [...] pra que a gente possa pensar em reativar o VER-SUS e [...] eu acredito que em 2012 a gente realizou duas edições do VERSUS novamente aqui em Teresina (ENTREVISTA 1, Comissão organizadora Teresina).

Em 2012, as vivências do Projeto não aconteceram somente em Teresina, mas também em cidades do interior do Estado, como Parnaíba, Piripiri, Beneditinos e Picos (Lira Neto et al., 2013). Destaca-se o fato de que a organização continuava vinculada ao NESP/UFPI, não existindo, portanto, coletivos organizadores nas demais cidades, para as quais as vivências foram descentralizadas.

Assim, novos coletivos do VER-SUS no Piauí surgiram somente em 2014, com a formação do Coletivo Litoral Piauiense, situado em Parnaíba. Os membros da comissão organizadora tiveram a oportunidade de participar de uma vivência no sistema de saúde do município de Sobral, Ceará. Com a experiência adquirida, o grupo se fortaleceu e retomou o Projeto em Parnaíba com a vivência 2015. Nesse contexto, o VER-SUS não ficou restrito ao município de Parnaíba, mas foi expandido para todo o Piauí, uma vez que ocorreu a participação de estudantes de todo o Estado.

Em 2014, [...] foi constituído um Coletivo VER-SUS Parnaíba, que desenvolveu uma vivência profunda no Sistema Municipal de Saúde de Sobral. A vivência emergiu do Núcleo de Estudos de Saúde Pública de Parnaíba e de um Coletivo de Estudantes que desejavam vivenciar um processo de formação implicada com os pressupostos da Reforma Sanitária Brasileira e do Sistema Único de Saúde. Assim, o Coletivo VER-SUS Parnaíba tem atuado de forma protagonista em diversos cenários desenvolvendo um processo de formação política [...] (RELATÓRIO Comissão organizadora, Parnaíba).

Nesse sentindo, a construção histórica do VER-SUS evidencia seu compromisso com a reorientação da formação em saúde. Por conseguinte, é apontado como um caminho transformador, marcado pela crítica ao modelo de ensino tradicional, no qual parte dos graduandos da área da saúde não é estimulada a compreender o SUS. Assim, muitos usam o sistema para seu aprendizado técnico-científico, no entanto, não são formados para atuar nele de forma crítica e reflexiva (Ferreira, Lira-Neto, Vale \& Oliveira, 2015).

Com efeito, os obstáculos que se interpõem às universidades no processo de inserção dos alunos em todos os âmbitos do sistema de saúde geram distanciamentos na promoção de debates e reflexões críticas relacionadas aos saberes e práticas na atenção à saúde da população, não havendo um aprendizado com base na realidade sanitária (Guimarães et al., 2019).

Evidencia-se que a retomada do VER-SUS pelo Coletivo Litoral Piauiense estimulou o recomeço do Projeto no estado, agora com organização descentralizada, como é possível identificar nas falas a seguir.

Eu fui vivente no coletivo de Parnaíba [...] juntamente com dois colegas de Picos. Então, nós tivemos interesse de trazer ele aqui pra Picos, justamente com a intenção de poder fornecer pra outras pessoas a mesma experiência que nós tivemos lá em Parnaíba (Entrevista 1, Comissão Organizadora Picos)

Conseguimos montar um coletivo em Teresina, acho que o retorno do VER-SUS Teresina [...] foi no começo de 20 [...] que teve a edição. (ENTREVISTA 1, Comissão organizadora Teresina) 
Além da retomada do projeto em Teresina, Parnaíba e Picos, um novo coletivo foi formado em Floriano-PI, VERSUS Princesa do Sul, com a comissão organizadora composta por estudantes que participaram do Projeto em Picos e Parnaíba. Em 2016, aconteceu também a primeira edição do VER-SUS Princesa do Sul.

A proposta do VER-SUS em Floriano surgiu a partir da minha participação em Parnaíba como vivente e eu me senti bastante afetado com o projeto, foi uma coisa que modificou como visão enquanto estudante, enquanto pessoa, né? E a partir dessa experiência eu comecei a falar com alguns amigos sobre a experiência, como era o projeto e [...] que isso podia [...] modificar a minha visão enquanto futuro profissional e enquanto pessoa [...] ao levar [...] para Floriano eu consegui chamar a atenção de alguns alunos da Universidade [...] eu consegui contaminar outras pessoas a fazer com que elas pudessem pensar que a vinda do projeto VER-SUS pra Floriano, pudesse fortalecer [...] enquanto militância estudantil, social. (ENTREVISTA 2, Comissão organizadora Floriano)

Com as falas expressas nessa seção, foi possível delinear a evolução histórica do VER-SUS no Piauí, bem como algumas relações do Projeto com a formação em saúde. Todavia, evidencia-se que projeto, como dispositivo de ensino, promove o contato dos participantes com novos processos formativos forjados na inserção em distintos cenários de práticas em saúde, além do convite ao protagonismo que conduz à ação de cada participante agente ativo no seu processo de formação, assim como futuramente na sua atuação profissional (Ferreira et al., 2015; Lira Neto et al., 2013).

Destarte, apesar de o Projeto VER-SUS ser operacionalizado há 13 anos no Piauí, ainda se encontra em fase de consolidação. Nesse contexto, para uma melhor compreensão serão detalhadas, a seguir, as metas, os objetivos e os eixos do Projeto, bem como, a construção de um modelo lógico que permitirá um entendimento da sua organização e funcionamento.

\section{Identificação dos objetivos, Metas e Eixos de intervenção do VER-SUS}

O VER-SUS tem como meta colaborar com a formação de profissionais que contribuam com o fortalecimento do SUS e que sejam sensíveis e críticos às necessidades da população. Com a realização do Projeto, espera-se constituir novas relações de compromisso e de cooperação entre estudantes, gestores, instituições de ensino e movimentos sociais, com o ensejo de efetivar a integralidade da saúde e a educação significativa de profissionais (Fettermann et al., 2018; Maranhão \& Matos, 2017).

No que concerne aos objetivos, o projeto visa facilitar a compreensão do funcionamento do SUS, levando em consideração seus princípios e diretrizes, além de reafirmar a saúde como direito social, fortalecendo uma consciência sanitária no contexto universitário. Procura proporcionar aos participantes uma compreensão voltada à relação Estado/sociedade no âmbito do direito à saúde e do conceito ampliado de saúde. Além de provocar nos estudantes o compromisso ético-político com os processos de transformação do setor, refletindo acerca do seu papel enquanto agente construtor e modificador das práticas sociais (Lira Neto, 2013).

O Projeto apresenta os seguintes eixos: Aprendizagem significativa; Pedagogia problematizadora; Multiprofissionalidade, Interdisciplinaridade e Transdisciplinaridade. Sendo que, a aprendizagem significativa ocorre quando o aprendiz é capaz de receber novas informações e racionalizar, de maneira a construir uma interação entre o seu conhecimento prévio e a informação adquirida (Ribeiro, Cavalcante, Teófilo, Albuquerque \& Vasconcelos, 2016; Lima et al., 2019).

Em relação à pedagogia problematizadora, defende-se que o argumento de sua finalidade é a superação das desigualdades sociais por meio do desenvolvimento do nível de consciência da realidade e atuação transformadora. Observa-se que sua formulação ocorre por meio de grupos de discussões com auxílio da ação motivadora do facilitador, este deve 
permanecer no mesmo grau de importância dos educandos, com o objetivo de conhecer a realidade concreta, aproximar-se criticamente sem imposição ou memorização, mas pelo processo de compreensão, reflexão e crítica (Maroja, Almeida Junior \& Noronha, 2019).

No referente à multiprofissionalidade, à interdisciplinaridade e à transdisciplinaridade, pode-se afirmar que estas apresentam como característica similar a aproximação de diferentes disciplinas para a solução de problemas específicos. $\mathrm{Na}$ multidisciplinaridade há um somatório de métodos, na interdisciplinaridade as metodologias são compartilhadas gerando uma nova disciplina e na transdisciplinaridade as metodologias unificadoras são compartilhadas, contudo construídas mediante a articulação de métodos provenientes de diversas áreas do conhecimento, podendo originar novas disciplinas ou permanecer como zonas livres (Roquete, Amorim, Barbosa, Souza, \& Carvalho, 2012; Ribeiro et al., 2016).

As metas, objetivos e eixos aqui elencados têm como intuito possibilitar aprendizagem, a produção de conhecimento e a experimentação dos dispositivos de saúde, passando por percepções distintas, construções e agregações de valores, estabelecendo relações e vínculos, desenvolvimento de ações pedagógicas direcionadas para a educação permanente, o que transforma o VER-SUS em caráter individual e essencial, dificilmente sendo replicado na academia; gerando oportunidade única de uma formação contemplada por aspectos singulares nunca vistos nas universidades (Ribeiro et al., 2016; Lira Neto et al., 2013).

Como foi visto, o Projeto proporciona a aprendizagem mediante a vivência, do cotidiano de trabalho da rede de serviços e, por meio, de práticas multi e interdisciplinares. Os estudantes podem compreender e avaliar o seu funcionamento, e fazer o exercício de, como futuros profissionais, traçarem possibilidades de ação. Isso proporciona um grande crescimento pessoal e profissional para os estagiários no que concerne à reflexão acerca de uma assistência de maior qualidade, quando estes ingressarem no SUS ou no campo da saúde em geral (Ferreira et al., 2015). Por outro lado, instiga os docentes a promover reflexões críticas com base na realidade social, promovendo a produção de conhecimento. Ademais, produz tensionamentos práticos, tanto na atenção quanto na gestão, assim como nas próprias relações interpessoais com potencial de desencadear novos modos de produzir e gerir saúde, considerando-se, sobretudo, a participação social nas práticas sanitárias.

No Quadro 1, demonstra-se os usuários potenciais da avaliação do VER-SUS, os interesses e os possíveis usos dos achados da avaliação. Foram identificados os seguintes usuários da avaliação do Projeto VER-SUS: Instituições proponentes, Instituições de ensino, Gestores de saúde, Comissões organizadoras, Viventes e Facilitadores. Com isso, identificou-se as impressões sobre os usuários, os interesses, as parcerias institucionais estabelecidas na execução do projeto e no uso da avaliação. Verificou-se a pertinência das parcerias estabelecidas para o enfrentamento do problema. A adequação das parcerias levou em consideração a possibilidade de criação de situações favoráveis ao cumprimento dos objetivos do programa. Com base na identificação dos usuários potenciais da avaliação e do papel que cada um exerce no programa, pode-se perceber que as parcerias estabelecidas são coerentes com o tipo de problema a ser enfrentado. 
Quadro 1 - Matriz de usuários potenciais da avaliação do Projeto VER-SUS, Piauí. 2020.

\begin{tabular}{|c|c|c|c|}
\hline $\begin{array}{c}\text { Usuário potencial da } \\
\text { avaliação }\end{array}$ & Papel do programa & Interesse na avaliação & $\begin{array}{c}\text { Papel na utilização dos } \\
\text { achados }\end{array}$ \\
\hline $\begin{array}{l}\text { Representantes das } \\
\text { Instituições } \\
\text { proponentes }\end{array}$ & Gestão do projeto & $\begin{array}{l}\text { Obter informações em } \\
\text { relação à modelização do } \\
\text { programa e ao seu } \\
\text { processo de execução }\end{array}$ & $\begin{array}{l}\text { Nortear a gestão do projeto, } \\
\text { além de acompanhar a sua } \\
\text { implementação }\end{array}$ \\
\hline $\begin{array}{l}\text { Representantes das } \\
\text { Instituições de ensino }\end{array}$ & $\begin{array}{l}\text { Apoio na organização, } \\
\text { implementação do projeto } \\
\text { e auxílio nas atividades }\end{array}$ & $\begin{array}{l}\text { Levantar reflexões sobre } \\
\text { o processo de ensino- } \\
\text { aprendizagem durante a } \\
\text { vivência }\end{array}$ & $\begin{array}{l}\text { Contribuir com ajustes para } \\
\text { melhorar na dimensão } \\
\text { político-pedagógica } \\
\text { projeto com vistas a } \\
\text { qualificá-lo }\end{array}$ \\
\hline Gestores de saúde & Apoio na organização & $\begin{array}{l}\text { Levantar reflexões sobre } \\
\text { a organização do projeto }\end{array}$ & Contribuir para ajustes \\
\hline $\begin{array}{l}\text { Membros } \\
\text { Comissões } \\
\text { organizadoras }\end{array}$ & $\begin{array}{l}\text { Construção e organização } \\
\text { da vivência, planejamento } \\
\text { e auxílio na realização das } \\
\text { atividades, e intermediar o } \\
\text { contato com todos os } \\
\text { envolvidos no projeto }\end{array}$ & $\begin{array}{l}\text { Obter informações para } \\
\text { melhorar as vivências e } \\
\text { refletir sobre os } \\
\text { produtos, resultados e } \\
\text { metas do projeto }\end{array}$ & $\begin{array}{lll}\text { Contribuir } & \text { para } & \text { a } \\
\text { organização, qualificação e } & \text { etas do } \\
\text { alcance das metas do } & \\
\text { projeto. } & & \end{array}$ \\
\hline $\begin{array}{l}\text { Viventes } \\
\text { facilitadores }\end{array}$ & $\begin{array}{l}\text { Participar das atividades } \\
\text { projeto previstas pela } \\
\text { organização }\end{array}$ & $\begin{array}{l}\text { Levantar } \quad \text { reflexões } \\
\text { relacionadas à formação } \\
\text { em saúde e sobre a } \\
\text { organização do projeto }\end{array}$ & $\begin{array}{l}\text { Contribuir com as melhorias } \\
\text { das edições do projeto a } \\
\text { partir do que foi vivenciado }\end{array}$ \\
\hline
\end{tabular}

Fonte: Dados da pesquisa.

\section{Modelo Lógico do Projeto VER-SUS}

O modelo lógico retrata um quadro que contribui para que todos os envolvidos no projeto identifiquem com clareza a racionalidade da sua construção e compreendam melhor as dimensões de sua implementação (Dias et al., 2018; Vieira-daSilva, 2018). Com base na análise dos documentos e das entrevistas, construiu-se o modelo lógico do VER-SUS, no qual foram identificadas e apontadas as relações entre os componentes e atores para o Projeto atingir os objetivos propostos (Figura 1).

A construção do modelo lógico, desenvolvida por meio do Estudo de Avaliabilidade, foi realizada mediante uma análise detalhada de todas as questões intrínsecas a operacionalização do Projeto VER-SUS no estado do Piauí. Para tanto, levou-se em consideração todas as articulações entre os envolvidos na execução dele, o que tornou o processo mais desafiador, pois para que isso acontecesse precisou-se compreender a teoria do projeto, além disso, houve a necessidade de analisar criteriosamente as entrevistas com o objetivo de edificar um modelo que realmente contemplasse a lógica real do projeto.

O processo de elaboração dessa representação possibilitou que a racionalidade implícita do Projeto se tornasse explícita, o que leva a uma maior possibilidade de construção de acordos em relação aos seus componentes e aos resultados de uma avaliação. Explicitar o modelo lógico consiste em desvelar quais os limites ou debilidades de seus pressupostos, o que permite identificar onde melhores evidências devem ser buscadas (Leviton et al., 2010; Dias et al., 2018).

Por meio do modelo lógico do Projeto VER-SUS foi possível evidenciar cada um de seus componentes, insumos/recursos, atividades, produtos, resultados e impactos. Cada um desses itens exige atividades administrativas determinadas a assegurar um ajuste entre a teoria do projeto, os objetivos, os recursos, as atividades e o alvo da mudança. 
Figura 1 - Modelo Lógico do Projeto VER-SUS, Piauí. 2020.

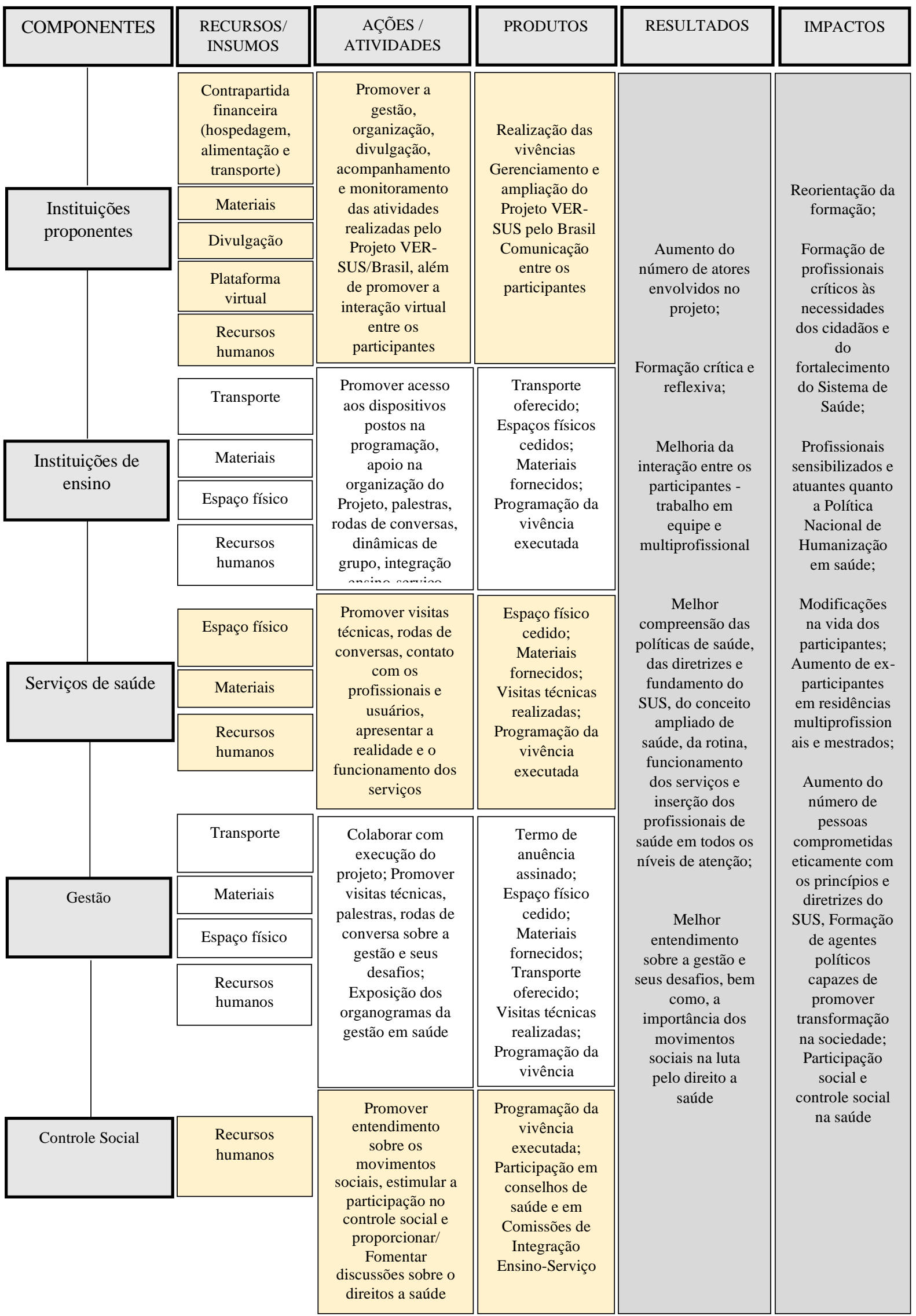

Fonte: Dados da pesquisa. 
Para elencar os componentes buscou-se conhecer a fundo toda a dinâmica, os envolvidos e a operacionalização do Projeto, com isso listou-se dentre os elementos o quadrilátero da formação em Saúde, que são as Instituições de Ensino, Serviços de Saúde, Gestão e Controle Social (Feuerwerker, 2014), além desses, acrescentou-se as instituições proponentes do projeto, para não ficarem lacunas na compreensão da sua execução e funcionalidade.

Em seguida, apontaram-se os recursos, insumos, atividades e produtos oferecidos por cada componente, sendo que os resultados e impactos foram listados de forma geral a partir de todos os componentes, pois na construção do projeto as atividades são organizadas em conjunto, ou seja, todos têm a sua importância e contribuem significativamente para o alcance dos objetivos propostos, em tempo, enfatiza-se que cada componente isoladamente não propicia/contempla todos os eixos estruturantes para uma aprendizagem significativa, além de não permitir um conhecimento ampliado do SUS e seus princípios, assim como não conseguem visualizar na prática a sua operacionalização.

Em tempo, é válido apontar que após a construção do modelo lógico, ele foi encaminhado para alguns sujeitos da pesquisa para apreciação. Na oportunidade, após análise, estes apontaram um posicionamento crítico acerca do modelo, com isso chegou-se à versão final, apresentada na Figura 1. Enfatiza-se que sua descrição constitui uma etapa importante do EA. Esta fase da pesquisa foi simulada por uma representação da teoria do projeto como requerido pela análise lógica, pois é por meio da compreensão do projeto que se observou as condições necessárias para avaliar as experiências locorregionais.

\section{Considerações Finais}

Os resultados demonstraram que o VER-SUS é passível de avaliação, uma vez que o modelo lógico permitiu a identificação de dimensões que se constituem focos prioritários de processos avaliativos voltados a aferir sua efetividade, cujo problema principal é a formação de profissionais críticos e sensíveis às necessidades de saúde da população e ao fortalecimento do SUS.

Torna-se necessário uma avaliação do VER-SUS voltada à efetividade do Projeto no âmbito da formação em saúde, pois esta pesquisa só permite uma compreensão das suas proposições e da operacionalização por meio dos componentes envolvidos na organização.

\section{Referências}

Costa, D. A. S., Silva, R. F., Lima, V.V., \& Ribeiro, E. C. O. (2018). Diretrizes curriculares nacionais das profissões da saúde 2001-2004: análise à luz das teorias de desenvolvimento curricular. Interface - Comunicação, Saúde, Educação, 22(67), 1183-1195.

Dias, M. S. A., Oliveira, I. P., Silva, L. M. S., Vasconcelos, M. I. O., Machado, M. F. A. S., Forte, F. D. S., \& Silva, L. C. C. (2018). Política Nacional de Promoção da Saúde: um estudo de avaliabilidade em uma região de saúde no Brasil. Ciência \& Saúde Coletiva, 23(1), $103-114$.

Ferla, A. A., Ramos, A. S., \& Leal, M. B.(2013). A história do VER-SUS: um pouco sobre o conjunto das iniciativas que inspiraram o projeto VERSUS/Brasil. In Ferla, A. A., Ramos, A. S., Leal, M. B., \& Carvalho, M. S. (Orgs.). Caderno de textos. Coleção VER-SUS Brasil. Rede Unida.

Ferreira, B. O., Lira-Neto, J. C. G., Vale, A. R., \& Oliveira, L. C.(2015). Uma (re)leitura de estagiários do VER-SUS/Piauí acerca do Sistema Único de Saúde. Rev. APS, 18 (2), 256 - 259

Fettermann, F. A., Nietsche, E. A., Terra, M. G., Salbego, C., Torres, O. M., \& Ramos, T. K. (2018). Projeto VER-SUS: Influências na formação e atuação do enfermeiro. Revista Brasileira de Enfermagem, 71(6), 3097-3104.

Feuerwerker, L. C. M.(2014). Micropolítica e saúde: produção do cuidado, gestão e formação. Rede Unida.

Guimarães, J. M. X., Garcia Filho, C., Ruiz, E. M. (Orgs.). (2019). Ensino na saúde: política, currículo e práticas na reorientação da formação. EdUECE.

Leviton, L. C., Khan, L. K., Rog, D., Dawkins, N., \& Cotton, D. (2010). Evaluability Assessment to Improve Public Health Policies, Programs and Practices. Annual Review of Public Health, 31 (1): 213-233.

Lima, V. S., Azevedo, N. A. A., Guimarães, J. M. X., Pereira, M. M., Agostinho Neto, J., Souza, L. M., Pequeno, A. M. C., \& Sousa, M. S. (2019). Produção de vídeo educacional: estratégia de formação docente para o ensino na saúde. RECIIS: Rev. Eletron. Comun. Inf. Inov. Saúde, 13(2), $428-438$.

Lira-Neto, J. C. G., Freitas, R. W. J. F., Brito, E. C. C., Santos, L. R., Alves, L.P. A., \& Alves, L. R. A. (2013). VER-SUS: um relato de experiência sobre uma vivência-estágio na realidade do Sistema Único de Saúde. Revista de Enfermagem UFPE, 7 (3), 1042-1046. 
Research, Society and Development, v. 10, n. 3, e56110313781, 2021

(CC BY 4.0) | ISSN 2525-3409 | DOI: http://dx.doi.org/10.33448/rsd-v10i3.13781

Maranhão, T., \& Matos, I. B.(2017). Vivências no Sistema Único de Saúde (SUS) como marcadoras de acontecimento no campo da Saúde Coletiva. Interface - Comunicação, Saúde, Educação, 22(64), 55-66.

Maroja, M. C. S., Almeida Júnior, J. J., \& Noronha, C. A. (2020). Os desafios da formação problematizadora para profissionais de saúde em um programa de residência multiprofissional. Interface - Comunicação, Saúde, Educação, 24, e180616.

Mendes, F. M. S., Fonseca, K. A., Brasil, J. A., Dalbello-Araújo, M. (2012). VER-SUS: Relato de Vivências na Formação de Psicologia. Psicologia: ciência e profissão, 32 (1), 174-187.

Mendes, T. M. C., Ferreira, T. L. S., Carvalho, Y. M., Silva, L. G., Souza, C. M. C. L., \& Andrade, F.B. (2020). Contribuições e desafios da integração ensinoserviço-comunidade. Texto \& Contexto - Enfermagem, 29, e20180333.

Minayo, M. C. S. (2014). O desafio do conhecimento: pesquisa qualitativa em saúde (14a ed.). Hucitec.

Moraes, B. A., Vieira, M. S. N., \& Campos, N. M. S.(2017). A influência de Programas de Reorientação da Formação em cursos da área da Saúde. Demetra: alimentação, nutrição \& saúde, 12 (3), 623-636.

Pinheiro, R., \& Ceccim, R. B. (2011). Experienciação, formação, cuidado e conhecimento em saúde: articulando concepções, percepções, e sensações para efetivar o ensino da integralidade. In Pinheiro, R., Ceccim, R.B., \& Mattos, R.A. (Orgs.). Ensinar Saúde: a integralidade e o SUS nos cursos de graduação na área da saúde. CEPESC: IMS/UERJ: ABRASCO.

Ribeiro, M. A., Cavalcante, A. S. P., Teófilo, F. K. S., Albuquerque, I. M. N., Vasconcelos, M. I. O. (2016). Vivências e Estágios na Realidade do Sistema Único de Saúde (VER-SUS) como agente promotor de mudanças na formação de graduação e nas práticas profissionais. Saúde \& Transformação Social, 7(1), 109.-119.

Roquete, F. F., Amorim, M. M. A., Barbosa, S. P., Souza, D. C. M., \& Carvalho, D. C. (2012). Multidisciplinaridade, interdisciplinaridade e transdisciplinaridade: em busca de diálogo entre saberes no campo da saúde coletiva. Revista de Enfermagem do Centro Oeste Mineiro, 2 (3), $463-474$.

Stella, R. C. R., \& Puccini, R. F.(2008). A formação profissional no contexto das Diretrizes Curriculares Nacionais para o curso de medicina. In Puccini, R.F., Sampaio, L. O., \& Batista, N. A. (Orgs.). A formação médica na Unifesp: excelência e compromisso social. Editora Unifesp.

Thurston, W. E., \& Ramaliu, A. (2005). Evaluability Assessment of a survivors of torture program: lesson learned. The Canadian Journal of Program Evaluation, 20 (2), 1-25.

Vendruscolo, C., Prado, M. L., \& Kleba, M. E. (2016). Integração Ensino-Serviço no âmbito do Programa Nacional de Reorientação da Formação Profissional em Saúde. Ciência \& Saúde Coletiva, 21(9), 2949-2960.

Vieira-da-Silva, L. M. (2018). Avaliação de políticas e programas de saúde. Fiocruz. 